\title{
Latent inhibition measured by heart rate suppression in rats
}

\author{
TIMOTHY K. WITTMAN and TERRY L. DeVIETTI \\ Central Washington University, Ellensburg, Washington 98926
}

In a latent inhibition paradigm, rats were given preexposure to a to-be-conditioned stimulus, a tone, and then were subsequently fear conditioned by a single pairing of the tone with footshock. Heart rate was recorded during the preexposure tones and at equivalent times for animals not given the preexposure tones. In addition, heart rate was recorded in both groups in response to the tone following conditioning. Results indicated that rats given preexposure to the tone showed a decrease in heart rate in the initial preexposure trial that returned to baseline across subsequent daily sessions. In the test, latent inhibition was demonstrated, as these rats showed less suppression of heart rate than did rats not given the preexposure tones. These results suggest that heart rate parallels the common skeletal measures of latent inhibition in the test and provide evidence that the impact of the to-be-conditioned stimulus is attenuated during the preexposure phase of the latent inhibition procedure.

Latent inhibition, the decremental effect of nonreinforced preexposure to a to-be-conditioned stimulus on subsequent conditioning/retention performance, has been shown to occur across a wide variety of behavioral tasks and species (Lubow, 1973). We have consistently obtained latent inhibition with six presentations of a tone preexposure stimulus, using a fear conditioning task and a drink latency measure of retention (DeVietti, Wittman, \& Comfort, 1980; DeVietti, Wittman, Emmerson, \& Thacher, in press). To the best of our knowledge, there have been no investigations examining the autonomic correlates of latent inhibition in experimental animals. A measure of autonomic activity during preexposure trials would seem to be especially useful in the development and elaboration of theoretical models of latent inhibition.

The present investigation was conducted as a first step in this direction. The experiment was essentially a replication of DeVietti et al. (1980), but with the following modifications: (1) Animals were not implanted with intracranial electrodes, and (2) heart rate reactivity served as the response measure.

\section{METHOD}

Subjects were 15 experimentally naive male Long-Evans rats, approximately 120 days old at the start of the experiment, selected from the Central Washington University animal colony. Each animal was anesthetized lightly with $\mathrm{CO}_{2}$ and fitted with two safety pins (No. 1), inserted subcutaneously on each side of the thoracic cavity, for the recording of heart rate. Twentyfour hours later, animals were handled by the experimenter and fitted with the heart rate recording harness. Adaptation to the harness was accomplished by allowing each animal to wear the

This research was supported in part by the small grants program of Central Washington University. Requests for reprints should be sent to Terry L. DeVietti, Department of Psychology, Central Washington University, Ellensburg, Washington 98926. device $5 \mathrm{~min} /$ day for 2 days. The harness was made of Plexiglas and fitted over the back of the animal. An elastic strap attached to one side of the harness was drawn ventrally beneath the forearms of the animal and secured on the opposite side to a Velcro patch. Alligator clips, soldered to wire leads, and a female connector, embedded in the harness, were attached to the safety pins.

Twenty-four hours following adaptation to the harness, animals were randomly assigned to either the preexposure (PE) or no-preexposure (NPE) condition. The preexposure stimulus consisted of a $15-\mathrm{sec}$ tone $(1,200 \mathrm{~Hz} ; 92 \mathrm{~dB})$ presented at Minutes 2 and 7 of each daily 10-min session, for 3 consecutive days. These trials were administered in a Plexiglas box with a grid floor, enclosed in a fan-ventilated soundattenuated chamber. Specific features of the apparatus have been described in detail elsewhere (DeVietti \& Larson, 1971). Individual animals were placed in the apparatus, with the harness and recording cable attached. Heart rate recordings were taken $60 \mathrm{sec}$ prior to the onset of each tone, during the tone, and for $60 \mathrm{sec}$ following the offset of each tone. Animals in the NPE condition were treated identically, except the tone was not presented during the preexposure phase of the experiment. Heart rate, recorded from the pins attached to the thoracic cavity, was amplified by an EKG polygraph recorder (Gilson, Inc.) onto a strip chart set at a constant speed of $10 \mathrm{~mm} / \mathrm{sec}$ Heart rate recordings were counted manually at the conclusion of the experiment by one of the authors, who was naive with respect to treatment condition for individual animals.

Following the last preexposure session, animals were water deprived in anticipation of drink training, to be initiated $24 \mathrm{~h}$ later. This procedure, as well as water deprivation prior to the test session, was included in the present investigation to equate conditions with the procedure used to obtain a lick suppression measure of latent inhibition (e.g., DeVietti et al., 1980). Drink training was accomplished by placing individual animals in the chamber, void of harness and cable, and allowing them to complete 110 licks from a tube inserted through one wall of the chamber. Following drink training, each animal was returned to the home cage, in which water was again made available.

Twenty-four hours after drink training, animals were given one-trial fear conditioning. Each animal was placed in the chamber, void of harness and cable, for an interval of $2 \mathrm{~min}$. At the end of $2 \mathrm{~min}$, training was initiated by presentation of the 15 -sec preexposure tone. Grid shock $(3 \mathrm{sec}, 1.6 \mathrm{~mA}, 60 \mathrm{~Hz}$, $330 \mathrm{~V} \mathrm{rms}$ ) was presented during the last $3 \mathrm{sec}$ of the tone. Each 
animal was removed from the apparatus $33 \mathrm{sec}$ later and returned to the home cage.

Twenty-four hours after training, animals were again deprived of water. In the test, $24 \mathrm{~h}$ later, each animal was placed in the apparatus with the recording harness and cable attached. The tone was presented by the experimenter $2 \mathrm{~min}$ into the session. Heart rate was recorded for $60 \mathrm{sec}$ prior to the tone, during the 15 -sec tone, and for $60 \mathrm{sec}$ following the offset of the tone. At the conclusion of the test, each animal was returned to the home cage, in which water was again made available.

\section{RESULTS}

Of the 15 animals employed in the present investigation, 2 rats from the PE condition and 1 rat from the NPE group were discarded because portions of the heart rate recordings were uninterpretable. A total of 12 animals, 6 in the PE condition and 6 in the NPE group, provided data for analysis.

Initial analysis of the heart rate prior to presentation of the tone in the PE group, and at the same intervals in the NPE group, across the preexposure days showed that heart rate decreased in both groups between the two baseline measures taken each day $[\mathrm{F}(1,46)=6.47$, $p<.05]$. No other source of variance approached statistical significance.

Given the absence of a significant interaction between prior exposure treatment and baseline heart rate activity, the raw data were transformed to suppression ratios (DeVietti \& Porter, 1969; Wittman \& DeVietti, 1981) for subsequent analyses. This transformation compares heart rate activity during CS presentation with heart rate activity occurring prior to stimulus introduction (baseline measure). In the present analysis, response to tone/no-tone presentation was determined by comparing heart rate for the first 5-sec block within the CS interval with the last 5 -sec interval of each of two 15 -sec blocks immediately preceding tone/no-tone presentation. For example, a suppression ratio calculated for the initial 5-sec block of the 15-sec tone presentation interval would by given by $2 \mathrm{CS} /\left(2 \mathrm{CS}+\mathrm{B}_{1}+\mathrm{B}_{2}\right) \times 100$, where CS refers to the sum of the heart beats for the first 5-sec target interval during tone presentation and $B_{1}$ and $B_{2}$ refer to the sums of heart beats counted during the last $5 \mathrm{sec}$ of two preceding baseline intervals. Absence of change in heart rate activity at the CS interval would be expressed by a suppression ratio of 50 , bradycardia would be shown by a value less than 50 , and a suppression ratio greater than 50 would indicate tachycardia.

In the analysis of the suppression ratios, we used only the performance on the first trial of each preexposure day in order to facilitate comparisons with the single test trial measure. This decision was justified on the grounds that a preliminary analysis revealed no differences in response to the two presentations of the tone within preexposure sessions.

Heart rate activity in response to the first tone presentation on preexposure days and in the test session was analyzed with a 2 by 4 split-plot analysis of variance, with groups (PE/NPE) as the between-subjects factor and days $\left(D_{1}, D_{2}, D_{3}\right.$, test) and interactions between these factors as within-subjects sources of variance. Results of this analysis revealed significant effects for days $[F(3,30)=13.21, p<.01]$ and Groups by Days $[F(3,30)=4.24, p<.02]$. The main effect of group failed to reach statistical significance $(p>.05)$.

The Groups by Days interaction is shown in Figure 1. The analysis of the breakdown of the simple main effects and subsequent $t$ tests, when appropriate, revealed that the PE group showed suppression of heart rate to the tone, relative to the NPE group, on Day 1 of preexposure $(p<.05)$. In the test, the PE group suppressed less to the tone than did the NPE group $(p<.01)$, thus demonstrating latent inhibition. The two groups did not differ on either Preexposure Day 2 or 3 (ps $>.05$ ). The pattern of heart rate activity across the 4 recorded days was quite different between the two groups. In the NPE group, heart rate suppression in the test was reliably greater, relative to the 3 preexposure days (ps $<.01$ ), and no significant differences were detected in the comparisons among the 3 preexposure days ( $p s>.05$ ). In contrast, the heart rate suppression in Group PE was greater on Preexposure Day 1 relative to Day $3(\mathrm{p}<.05$, one-tailed test), but no difference between performance on preexposure days was discernible ( $\mathrm{ps}>.05$ ). In the test, the suppression of the PE group was greater than that obtained on Days 2 and 3 (ps $<.05$ ), but it did not differ from the suppression obtained on Day $1(\mathrm{p}>.05)$.

\section{DISCUSSION}

The present experiment demonstrated that rats given preexposure trials in a latent inhibition paradigm initially reacted with a decrease in heart rate to the to-be-conditioned stimulus

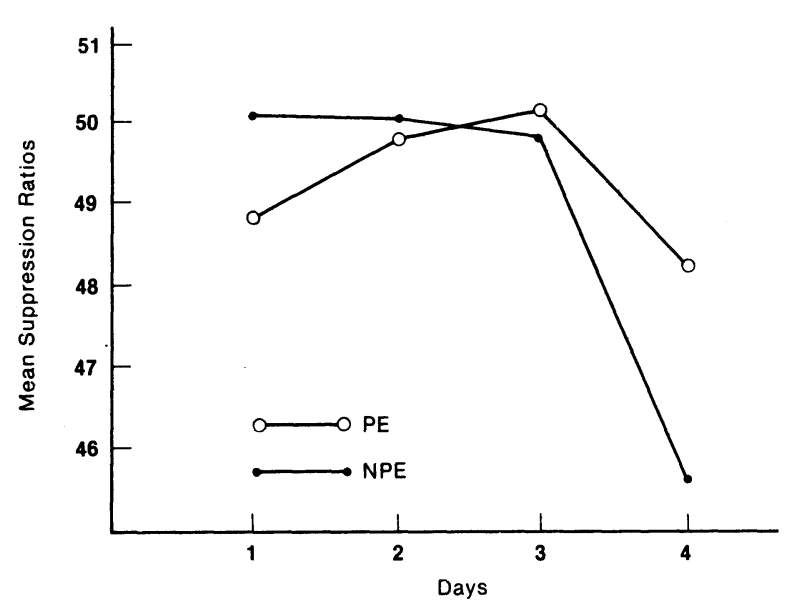

Figure 1. Mean heart rate suppression ratios across 3 preexposure days and the test. 
and that this response attenuated across daily preexposure trials. Further, following conditioning, rats given preexposure trials showed latent inhibition by decreasing heart rate less to the test stimulus, relative to rats not given preexposure trials

A pervasive theme in recent explanations of the latent inhibition phenomenon is that the redundancy and irrelevance of the preexposed stimulus is central to the effect. For example, it has been suggested that the organism learns to "tune out" (Solomon \& Moore, 1975) or "ignore" (MacKintosh, 1975) the preexposure stimulus as a result of its irrelevance. Lubow, Schnur, and Rifkin (1976) suggest that the initial presentations of the to-be-conditioned stimulus produce an attentional response in the organism. However, in the absence of a second stimulus (e.g., an unconditioned stimulus), this attentional response dissipates with repeated presentations. All of these notions receive a measure of support in studies using conventional behavioral measures, such as the development of subsequent conditioning and/or retention of conditioning (e.g., DeVietti et al., 1980; Lubow et al., 1976; Solomon \& Moore, 1975). However, as such measures are taken after preexposure treatment, any speculation as to the effects of the to-be-conditioned stimulus prior to conditioning must be indirect. In the present experiment, however, the recording of heart rate during the preexposure trials enabled us to evaluate directly the impact of the to-be-conditioned stimulus, and the results supported the idea that the initial impact of the to-be-conditioned stimulus is reduced as a consequence of repeated preexposure trials. We suggest that the use of autonomic measures, during both the preexposure phase and the conditioning/retention phases of latent inhibition experiments, may be useful in the development of models to explain the phenomenon.

\section{REFERENCES}

DeVietti, T. L., \& Larson, R. C. ECS effects: Evidence supporting state-dependent learning in rats. Journal of Comparative and Physiological Psychology, 1971, 74, 407-415.

DeVietti, T. L., \& Porter, P. B. Modification of the autonomic component of the conditioned emotional response. Psychological Reports, 1969, 24, 951-958.

DeVietti, T. L., Wittman, T. K., \& Comfort, M. K. Rapid development of latent inhibition: No effect of implanted site. Psychological Reports, 1980, 47, 473-474.

DeVieTti, T. L. Wittman, T. K., Emmerson, R. Y., \& Thacher, D. O. Stimulation of the mesencephalic reticular formation or a flashing light increases latent inhibition to a tone conditioned stimulus. Behavioral and Neural Biology, in press. Lubow, R. W. Latent inhibition. Psychological Bulletin, 1973, 79, 398-407.

Lubow, R. E., Schnur, P., \& Rifkin, B. Latent inhibition and conditioned attention theory. Journal of Experimental Psychology, 1976, 2, 163-174.

MacKintosh, N. J. A theory of attention: Variations in the associability of stimuli with reinforcement. Psychological Review, 1975, 82, 276-298.

Solomon, P. R., \& Moore, J. W. Latent inhibition and stimulus generalization of the classically conditioned nictitating membrane response in rabbits (Oryctolagus cuniculus) following dorsal hippocampal ablation. Journal of Comparative and Physiological Psychology, 1975, 89, 1192-1203.

Wittman, T. K., \& DeVietti, T. L. Autonomic reactivity to reminder treatment predicts test performance in rats given ECS after training. Physiological Psychology, 1981, 8, 515-521. 